\title{
Accepter le flambeau
}

\author{
par Stephen Shalansky
}

$\mathrm{D}_{\text {m }}^{\mathrm{n}}$ ans le numéro de mars-avril 2016 du Journal canadien de la pharmacie hospitalière (JCPH), $\mathrm{D}^{\text {re }}$ Mary Ensom annonçait qu'elle quittait son poste de rédactrice en chef et qu'elle avait procédé à " passer le flambeau ${ }^{1}$. Elle y a brièvement présenté les nombreux succès qu'elle et le Journal ont connus pendant ses 10 années à la barre. Environ au même moment, Peter Zed, le rédacteur en chef intérimaire, m’a approché pour me demander si le poste m’intéressait. Je n'ai pas eu à réfléchir longtemps à cette proposition, mais j'étais tout de même intimidé à l'idée de succéder à Mary.

C'est un réel privilège de travailler de nouveau à la publication de notre Journal (j’y ai été rédacteur adjoint par le passé). Je dis " notre Journal » parce que je considère les lecteurs, les auteurs, les rédacteurs et les réviseurs du JCPH comme une communauté très soudée présentant bon nombre de ressemblances professionnelles malgré les grandes distances qui séparent ces individus et la diversité de leurs feuilles de route. Linfluence que le JCPH a eue sur notre profession et l'immense expertise de l'équipe de rédaction m’ont convaincu de relever ce défi.

Et quelle équipe magnifique! Nous devrions tous apprécier l'impressionnante gamme de points de vue internationaux qu'incarne l'équipe de rédaction du JCPH. Au cours des dernières années, l'équipe s'est enrichie. Elle compte maintenant d'éminents chercheurs de différents pays dans le monde. Ainsi, les rédacteurs adjoints actuels représentent le Canada, les ÉtatsUnis, l'Écosse et l'Australie. En travaillant ensemble, les membres de cette équipe ont fait croître les ressources du Journal et ont facilité la publication d'une plus grande variété de manuscrits. Bien que le Journal continue de mettre l'accent sur les articles abordant la participation des pharmaciens d'hôpitaux canadiens aux soins des patients, son mandat s'est élargi. Ce dernier inclut des manuscrits qui sont éducatifs pour les pharmaciens dans d'autres milieux de soins de santé misant sur la collaboration et dans d'autres pays. Par exemple, la série actuelle du JCPH sur les systèmes de santé internationaux décrit le rôle de pharmaciens de partout dans le monde, ce qui permet aux pharmaciens du
Canada et d'ailleurs de connaître les innovations qui peuvent être utiles pour leurs propres pratiques.

Je suis très fier de contribuer au succès continu du Journal et je promets de travailler avec ardeur pour qu'il continue de faire des progrès. Je prends cette responsabilité au sérieux, car je crois sincèrement que le JCPH a eu un effet positif sur la carrière de bon nombre de pharmaciens et sur les soins quils procurent aux patients. Le comité de rédaction du Journal fera tous les efforts nécessaires pour continuer à publier de l'information qui vous aide, vous, les lecteurs, à prodiguer les meilleurs soins possibles aux patients. Nous nous efforcerons aussi de trouver des façons novatrices de diffuser de nouvelles informations précieuses pour l'évolution de notre profession. Mais, nous ne pouvons atteindre ce but que grâce à l'intérêt soutenu de notre lectorat, aux commentaires que vous émettez et aux manuscrits que vous nous soumettez. J'ai très hâte de travailler avec vous alors que je saisis le flambeau tendu par Mary et que je le porte sur la voie de l'avenir et du succès continu du JCPH.

[Traduction par l'éditeur]

\section{Référence}

1. Ensom MHH. Ouvrir la voie et passer le flambeau. Can J Hosp Pharm. 2016;69(2):106.

Stephen Shalansky, Pharm. D., ACPR, FCSHP, est coordonnateur clinique du service de pharmacie au Providence Health Care et professeur de clinique à la Faculté des sciences pharmaceutiques de l'Université de Colombie-Britannique, à Vancouver, en Colombie-Britannique. ॥ est également rédacteur en chef du Journal canadien de la pharmacie hospitalière.

Intérêts concurrents : Aucun déclaré.

Adresse de correspondance :

Dr Stephen Shalansky

Pharmacy Department

Providence Health Care

1081 Burrard Street

Vancouver BC V6Z1Y6

Courriel : sshalansky@providencehealth.bc.ca 